Article

\title{
Ecological Systems as Complex Systems: Challenges for an Emerging Science
}

\author{
Madhur Anand $^{1, *}$, Andrew Gonzalez ${ }^{2}$, Frédéric Guichard ${ }^{2}$, Jurek Kolasa ${ }^{3}$ and Lael Parrott ${ }^{4}$ \\ 1 School of Environmental Sciences, University of Guelph, Guelph, Ontario, N1G 2W1, Canada \\ 2 Department of Biology, McGill University, Montréal Quebec, H3A 1B1, Canada; \\ E-Mails: andrew.gonzalez@mcgill.ca (A.G.); fred.guichard@mcgill.ca (F.G.) \\ 3 Department of Biology, McMaster University, Hamilton, Ontario, L8S 4K1, Canada; \\ E-Mail: kolasa@mcmaster.ca \\ 4 Département de Géographie, Université de Montréal, Montréal, Quebec, H3C 3J7, Canada; \\ E-Mail: lael.parrott@umontreal.ca
}

* Author to whom correspondence should be addressed; E-Mail: manand@uoguelph.ca; Tel.: +1-519-824-4210; Fax: +1-519-837-0442.

Received: 30 December 2009; in revised form: 1 March 2010 / Accepted: 8 March 2010 / Published: 15 March 2010

\begin{abstract}
Complex systems science has contributed to our understanding of ecology in important areas such as food webs, patch dynamics and population fluctuations. This has been achieved through the use of simple measures that can capture the difference between order and disorder and simple models with local interactions that can generate surprising behaviour at larger scales. However, close examination reveals that commonly applied definitions of complexity fail to accommodate some key features of ecological systems, a fact that will limit the contribution of complex systems science to ecology. We highlight these features of ecological complexity — such as diversity, cross-scale interactions, memory and environmental variability-that continue to challenge classical complex systems science. Further advances in these areas will be necessary before complex systems science can be widely applied to understand the dynamics of ecological systems.
\end{abstract}

Keywords: complex systems science; ecological indicators; environmental variability; model 


\section{Introduction}

Since seminal publications on the subject [1,2], the study of "ecological complexity" has gained momentum. Examples of this trend can be found in recent books [3], national research efforts (USA National Science Foundation "biocomplexity” initiative), and international journals (e.g., Ecological Complexity). The field borrows tools and theories from research in complex systems science (CSS) to describe and understand ecological phenomena. However, despite growing recognition of the utility of CSS in many disciplines, the field of ecological complexity has yet to be widely adopted by ecologists and remains controversial to many. There is widespread consensus that ecological complexity emerges from the interactions between organisms and their biotic and abiotic environments, and attempts to clarify these basic notions have produced several useful conceptual definitions of ecological complexity [4,5]. For example Levin (1998) considers ecological systems to be the prototypical complex adaptive systems (CAS) "in which macroscopic system properties such as trophic structure, diversity-productivity relationships, and patterns of nutrient flux emerge from interactions among components, and may feed back to influence the subsequent development of those interactions". The CAS approach like all CSS approaches attempts to explain all ecological phenomena with a restricted set of rules and constraints. Although clear and operational (and widely cited) ecologists have yet to widely integrate this perspective within their methodology. We believe that much of the hesitancy surrounding CSS is caused by uncertainty about what the approach brings to ecology. Any new approach to studying ecological complexity should not only suggest new models and formalisms but also new interpretations of ecological phenomena. We show how CSS has contributed to three important research areas in ecology, namely, food webs, patch dynamics, and population fluctuations. In reviewing these examples, we point out the limitations of the existing definitions of ecological complexity derived from CSS and suggest new directions for improved integration between CSS and ecology.

\section{Contribution of CSS to Ecology}

\subsection{Simple Measures and Models}

Definitions of complexity and thus their measures are diverse [6-11], a symptom of an emerging and quickly evolving discipline. Most definitions (about 31; [12]) stem from information theory and reflect the belief that a system is complex when it comprises many different parts whose combined state or behavior are difficult to predict. These definitions thus often assign the highest degree of complexity to random states. Other similar examples of information theoretic definitions are (1) effective complexity that emphasizes the degree of "regularity" (rather than randomness) displayed by a system

(2) computational complexity with its several forms (algorithmic, Kolmogorov, grammatical, time complexity, and others) which all reflect the amount of effort needed to represent the system; and (3) mutual information - the degree to which states of system components are mutually predictive [14]. Measures based on these definitions of complexity often start with two extremes of random and ordered states, with the highest complexity occurring somewhere in between (Figure 1). Complex states can also be described by statistical scaling relationships such as fractal dimension or 
power-laws [15]. Simple measures such as these can be used to distinguish a random or homogenous ecological pattern from a complex one and may serve as diagnostic tools to detect changes in the state of an ecosystem [16], as we shall illustrate in subsequent sections.

Figure 1. Temporal, spatial and structural signatures of simple ordered and disordered systems versus a complex system. (a) Sinusoidal time series; ordered spatial pattern; regular network; (b) Population dynamics of a modelled plant species from the individual-based model WIST [38]; spatial distribution of prey in the Lotka-Volterra model with diffusion over a coupled map lattice (generated with the model in [39]); scale-free network typical of some food webs; (c) Uniformly distributed random noise; random spatial distribution; random network. For each case, the rightmost column shows a schema of a corresponding frequency analysis, one of the most common descriptive measures of complexity that can be applied to temporal, spatial or structural signatures. For each case (a, b, c), the analysis is shown for the distribution of frequencies in the temporal signature, although it might also be applied to measure the distribution of patch sizes in the spatial signature, or the distribution of links per node in the network.

(a)

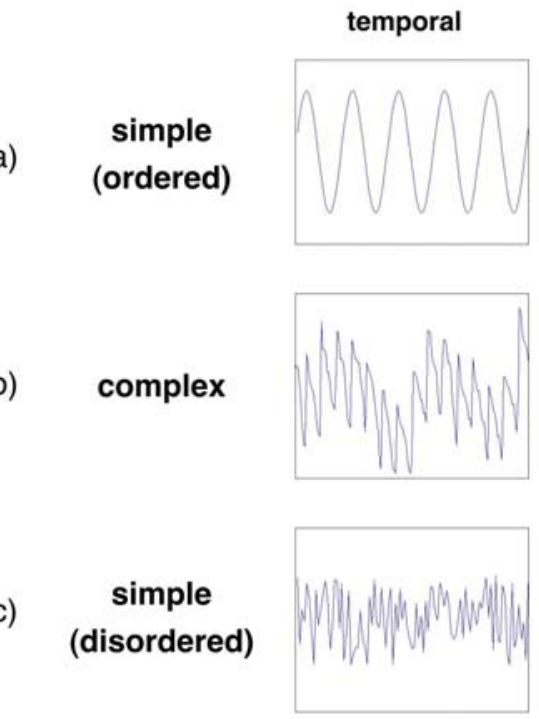

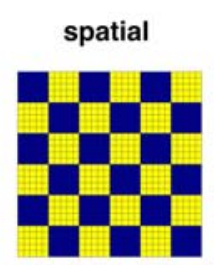
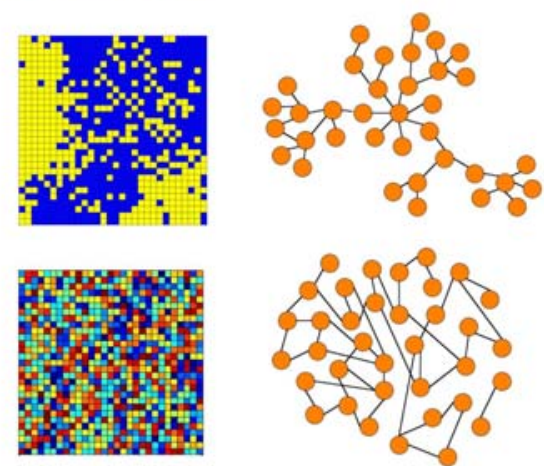
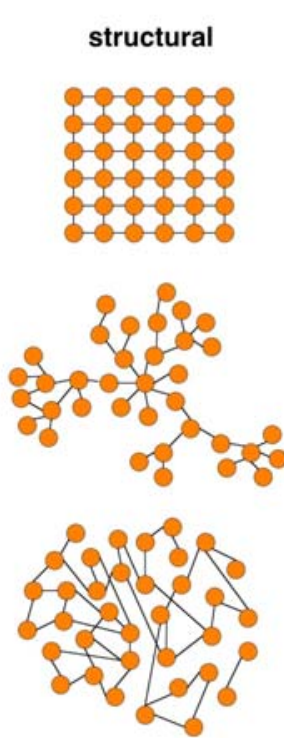
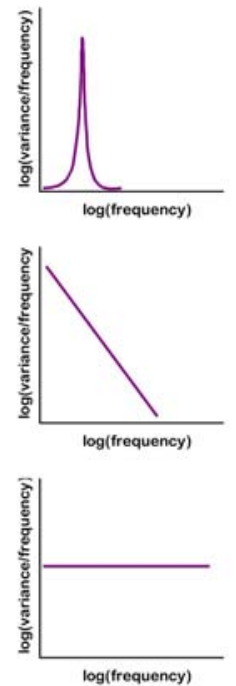

While such static descriptions and associated measures of complexity are important, they do not, in and of themselves, tell us how complex states come about. Towards this goal, null models must be used to first identify those complex patterns and structures emerging from purely random processes (see Figure 1c); a recent example is the prediction of many ecological patterns by neutral theory [17]. However, application of CSS to ecology requires that complex signatures distinguish random from non-random ecological processes. This can be seen by comparing Figure 1b and 1c, in particular the temporal, spatial or structural signatures shown in the last column. Thus, of interest to CSS are patterns and structures that arise from non-random processes. This points to another major contribution of CSS to ecology: the observation that simple models, with certain key non-random features, can generate surprisingly complex structures and dynamics. Ecologists have embraced this by modelling ecosystems as simplified systems of interacting particles (e.g., individuals, 'agents', species), often in 
well-defined, unchanging spatiotemporal environments. The use of simple models can improve our understanding of ecological dynamics and the mechanistic origins of observed patterns [18]. Classical models from CSS emphasize the importance of local interactions between the 'particles' for the formation of self-organized structure at larger scales [19], and indeed these types of models have been able to explain the existence of surprising ecological patterns [20-22].

In the following subsections, we elaborate on how simple measures and models from CSS have improved our understanding in three important areas of ecology, namely food webs, patch dynamics and population fluctuations.

\subsection{Food Webs}

Food web theories have traditionally emphasized the study of stability and complexity in relation to the structure of trophic interactions. Recent research in graph theory and networks has shown that many complex systems share common structural features. For example, diverse physical, biological and human-created systems have all shown scale-invariant structure, in which the network is organized around several large "hubs" with many less connected nodes [23] (Figure 1). This type of structure appears to be robust to the loss of system components and resilient to dynamic changes in node characteristics [24], implying stability-a feature of interest to biodiversity and ecological research. Quantitative methods used to study complex networks have migrated to food web research, leading to a resurgence of interest in food web structure and a re-analysis of many well-known data sets [25,26]. While there is debate about whether food webs have scale-free structures, the study of degree distributions (the distribution of the number of links per node) and other topological characteristics, has shown that food webs all have complex, non-random structures. New analytical methods have enabled ecologists to identify key aspects of food web structure, such as clusters of closely interacting species $[27,28]$ and the prediction of patterns of coextinction [29]. Such results are re-fueling the debate about whether ecological communities assemble towards structures that favor persistence and dynamic stability [30,31].

While the above research has contributed to the characterization of the static structures of food webs, new dynamic models of population dynamics from CSS have shed light on the study of how food web structures assemble and change over time [32-36]. These models reproduce many phenomena observed in empirical data such as power law scaling of speciation and extinction events, non-random (but not scale-free) network structure and relatively short food chain lengths. Similar to traditional Lotka-Volterra style population models, these models treat food webs as large evolving networks where each species is a node whose population size changes as a function of its interactions with other species. However, these new models borrow from recent methods in evolutionary computing to allow a species to adapt its interaction strengths with other species, thus dynamically changing the structure of the food web [37]. In these models, species are represented by digital “genomes”. Low levels of mutation during reproduction allow a species’ genome to evolve, enabling a species to adapt its interaction strengths and other parameters. Such dynamic models generate spatio-temporal data on interaction webs (not just predator-prey networks) over many generations, which would be difficult or impossible to measure in the field, leading to the generation of new hypothesis about how ecological communities are structured and assembled over time. In addition to 
creating new avenues of research in ecology, such as studies of species co-evolution and the formation of ecological communities, such models may enable ecologists to draw parallels between the dynamics of ecological networks and other similar complex biological systems (e.g., neuronal or biochemical networks).

\subsection{Patch Dynamics}

Diversity can be maintained through life-history trade-offs between growth rate and competitive ability. This is the prediction of the intermediate disturbance hypothesis, which was first formulated as a spatially homogeneous theory. In contrast to theories that assume 'well-mixed' disturbance, patch dynamics predicts species diversity in relation to the intensity and size of newly formed gaps [40-43]. It more precisely allows dispersal limitation to explicitly mediate species coexistence. Patch dynamics theory assumes independence between processes of gap formation and community recovery, and the apparent separation of scales between physical disturbances and population growth in various ecosystems has thus motivated its adoption in ecology. However, many disturbed systems, including forest fires involve direct and local feedbacks between disturbance and recovery. CSS has been applied to those cases using dynamic models that define all processes at scales close to those of individual organisms. These models contrast with earlier patch dynamic models by defining processes of disturbance and recovery as local (i.e., between neighbors) and spatially explicit. More importantly, these models conform to the general framework of CSS by recognizing the potential for such local processes to explain and generate macroscopic properties of disturbed ecological systems. More precisely, CSS models of disturbance dynamics predict the self-organization of long-range correlation between (distant) individuals, as supported by scale-invariant (i.e., power law) frequency distributions of patch sizes.

Large-scale properties of disturbance regimes become predictions that are solely based on local rates and geometry of disturbance and recovery. One important implication is found in the form of threshold responses to environmental and biological conditions. For example, the scale-invariant frequency distribution of gap sizes observed in some tropical forests can be predicted by the simple domino effect of wind throws, a process similar to the interactions between particles in physical systems [44,45]. Rocky intertidal communities provide additional examples [46,47]. CSS has thus resulted in a distinctive and useful theory of disturbance dynamics associated with testable predictions that can still be linked to patch dynamics [48]. However, associating data with specific models is still problematic. One problem comes from the fact that scale-invariant distributions have alternative causes, which fail to support scale-invariance as a signature of self-organization [49]. The sensitivity of simplistic CSS models to species interactions and to environmental fluctuations is also largely unknown.

\subsection{Population Fluctuations}

The classic question in population ecology of the effects of density dependence and of environmental fluctuations on population regulation has also benefited from CSS analysis. Time series of population dynamics were recently analyzed for signatures of ecological complexity (Figure 1). In particular, these analyses have addressed whether the observation of "more time means more 
variation" in population densities [50,51] is a signature of a power law relationship between the frequency and the variance of population fluctuations. More specifically, population fluctuations with a $1 / f^{\beta}$ pattern have a variance (power) that is proportional to the reciprocal of frequency ( $f$ ); the dominance of low frequencies engenders the property of increasing variance and a long-term autocorrelation in densities over time. In an analysis of 544 long-term population time series Inchausti and Halley found that the distribution of spectral exponents centered on a median value of 1.0 indicating that many populations have a $1 / f$ distribution [52]. CSS has paid special attention to $1 / f^{\beta}$ fluctuations, where beta is equal to one because this is indicative of scale invariance, a property typical of many complex phenomena like phase transitions that have fluctuations that decay as power laws. Unfortunately, the discovery of $1 / f$ fluctuations in nonequilibrium systems, like populations, is not evidence for any particular model of how the world works, such as self-organized criticality [53,54]. However, the widespread incidence of $1 / f$ population fluctuations is of genuine interest to population biologists because it pertains to the long-standing issue of population regulation and density dependence.

Standard ecological models based on logistic growth suggest tight density regulation and stationarity (no change) in the mean and variance of population densities. A $1 / f$ pattern in population abundances is evidence against stationarity and a simple view of population regulation in a fluctuating environment [52,55]. Additionally, it has been shown that the power spectra of chaotic dynamics produced by classic population models were dominated by high frequency fluctuations (blue spectra), a direct contradiction with the time series analyses [56]. Population biologists were stimulated to address these apparent mismatches between the power spectra of population time series and those produced by standard population models because they raise fundamental questions about our understanding of the cause of population fluctuations [57]. Several alterations to the standard population models were proposed to create strong low frequency variation (reddened spectra) in their dynamics [58,59]. For example, delayed density dependence may redden population dynamics by dampening high frequency variation in density [60]. Alternatively, local dispersal in spatially explicit metapopulations may also redden population dynamics; here local dispersal slows the rate of spread (impact) of local population lows so that low frequency variation dominates [61]. These theoretical results and advances were the direct result of a CSS approach to the analysis of population time series. The widespread presence of $1 / f$ dynamics is not unique to ecological systems but with the integration of CSS tools and methods population ecologists were able to directly tackle and provide alternate explanations for this intriguing phenomenon.

\section{Contemporary Research in Ecological Complexity}

\subsection{Integrating Classical CSS with Ecological Complexity}

We believe that measures and models from classical CSS will have limited applicability for ecology in the future because ecologists' perception of complexity invariably includes diversity, interactions that cross many spatial, temporal and organizational scales, ecological memory (historical effects), and heterogeneous and fluctuating environments. We illustrate the generic components of ecological complexity with an example of a forest ecosystem (Figure 2). Ecological memory is a record of past events that may introduce a temporal dependence in the dynamics of the system because it influences 
current and future states. We emphasize the importance of memory and environmental variability because these are key features of ecological systems that create and maintain diversity (genetic and phenotypic). Diversity is not just a passive effect of ecological interactions, but an important determinant of a system's persistence and adaptability in the face of environmental change. Environmental variability is ubiquitous; nevertheless debate continues over the priority of internal versus external causes of ecological variability [62]. A synthesis of these two perspectives acknowledges the possibility that ecological complexity might be generated by the interaction between a variable environment and internal self-organized dynamics, an aspect notably lacking from standard definitions of complex systems that typically exclude environmental (external) effects. Some of these aspects of ecological complexity are not easily accommodated by the current methodology and tools offered by CSS, however emerging research in the field is beginning to offer solutions.

\subsection{New Measures: From Diversity to Complexity}

Ecologists have widely adopted information-theoretic definitions from CSS to measure diversity (e.g., Shannon entropy), but few have attempted to quantify complexity based on other information and coding theoretic definitions proposed by CSS. The latter, which include adoption of concepts of algorithmic complexity [63-66] and mean information gain [66], could help to integrate concepts such as cross-scale interactions and change into static measures of complexity. New measures need to also integrate existing statistical descriptors of variance (or invariance) and disorder (or order) and be more explicitly linked to dynamic ecological concepts such as disturbance and stability. This may help to resolve the fact that the unpredictability (high disorder) associated with high Shannon diversity values seems at odds with the role of diversity in the maintenance of stability (predictability) in ecosystems [67]. The integration of environmental variability, cross-scale interaction and memory, and key features of complexity into ecological indicators, remains a challenge in ecology. Cross-scale integration of diversity and complexity measures can be achieved by linking scale-invariance of species-area curves and species abundance distributions with various diversity indices via multifractal analyses [68]. Similarly, integration of spatial variability and classical disorder-based diversity in a measure of complexity has been shown to capture cross-scale patterns [69]. Others [70] have introduced a complexity measure to characterize the boundary between ordered and disordered phases (in random Boolean networks) that incorporates variance. This approach may provide a solution to the recent call for connectivity measures to incorporate "matrix" effects (spatiotemporal heterogeneity) on metapopulation and landscape complexity [71]. A promising measure of complexity reflecting the dynamics of diversity (dynamical entropy), that explicitly examine the relationships between diversity, complexity, perturbation and stability has also been recently proposed [72]. Interestingly, a new measure founded in hierarchy theory [55] finds that highest levels of complexity occur at intermediate degrees of integration and low levels of perturbation. A number of other indicators have been proposed, including increasing variance and increasing autocorrelation in key system variables prior to collapse [73]. New measures which attempt to integrate disturbance and variability, into classical measures of complexity, will be of particular diagnostic value in studies of global ecological change. Furthermore, approaches that provide further understanding on the links between structural (static) and dynamical complexity will be useful [74,75]. 
Figure 2. An illustration of the components of ecological complexity. Complex ecological systems (e.g., forests) are those in which structure (e.g., gap-size distribution) and dynamics (e.g., succession) derive from memory-based (e.g., previous tree falls, seed bank), cross-scale (e.g., 100 sq. m to 100000 sq. m), interactions between diverse selfreproducing elements (e.g., individuals of different tree species) and their variable environment (e.g., climatic fluctuations). Current CSS models of scale-invariance of gap size distribution in forests only incorporate local interactions between trees and gapformation is attributed to chance environmental events, such as a wind throw (Panel A). Gap propagation, however, may be intricately linked to environmental variables (e.g., climate, soil) that cause gap sizes to occur with frequency distributions other than random (see Figure 1). For example, stochastic gap-creation due to wind throw may be amplified through feedbacks between gap size, fetch and boundary effects (Panel B) [45]. Recovery potential of forests within gaps may be dependent on memory-based spatiotemporal processes such as the presence of seed banks (Panel C). In addition, some tree species may be inherently more resistant to gap-formation than others due to their growth patterns. These types of diversity, memory, cross-scale interactions and environmental variability need to be incorporated into new models of ecological complexity.

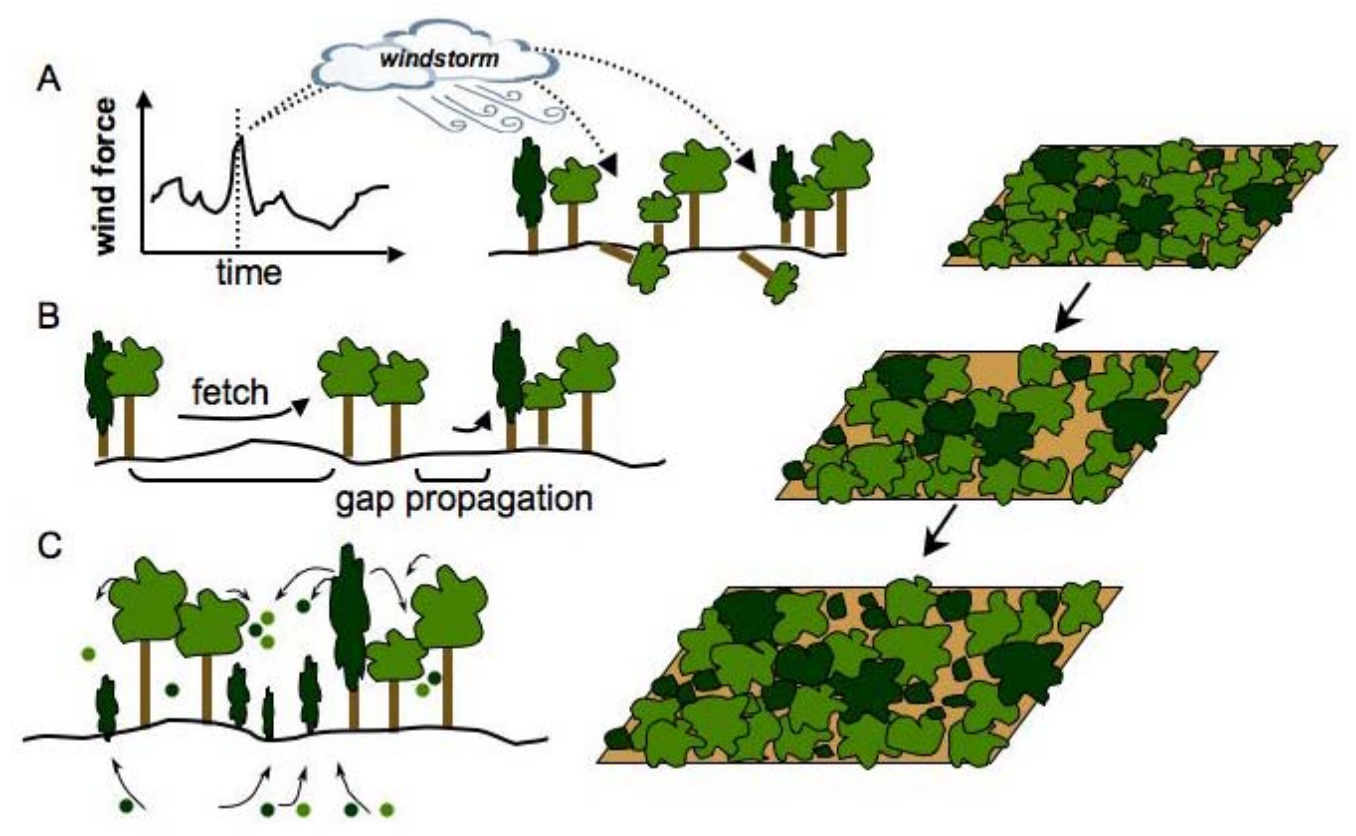

\subsection{The Role of Memory}

Ecological memory is invoked by the capacity of past states to influence present or future responses of an ecological system [76]. Examples include diapause eggs, seed banks, and the existence of rare species in an ecosystem that may represent the record of previous growth or species invasions [77,78]. Disturbance (fire, drought) may create highly persistent patterns at the landscape scale that may entrain future ecosystem processes [76,79,80]. Memory implies that the system can draw upon past events to inform current and future behaviour; this is typically the property of the organism. For example, recent results suggest that organisms can enhance their fitness by drawing upon the memory of past prey 
quality, or distribution, when foraging [81,82]. From an observer's perspective, memory is manifested by a strong temporal dependence in the dynamics of an ecological system [83]. Modeling memory-based systems can be achieved by incorporating autocorrelation or a time-lag into the model structure [84], but it remains a challenge yet to be addressed by many CSS models. The trend in individual-based models [85] (IBM) is to incorporate memory by allowing "agents" to make predictions based on past information; such predictions may be cue-based and hence relevant to many ecological systems, or may allow "agent” behaviour to be adaptive and heritable invoking evolution. These and other approaches could redefine the phenomenology of complex systems where localized interactions rely on recorded past events to operate across spatiotemporal scales.

\subsection{Complex Effects of Environmental Variability}

An important characteristic of ecological systems is the incessant environmental variation to which they are exposed; yet most CSS models used in ecology do not consider its effect. Two important questions immediately come to mind: To what extent are results from CSS models of population and community dynamics robust to environmental variability? Can the interaction of CSS with variable environments lead to novel dynamics? A good example of the importance of these questions is illustrated in the studies of complex spatial dynamics of host-parasitoid coexistence [86]. The Nicholson and Bailey formulation of host-parasitoid interactions is notoriously unstable in homogeneous space. Using a coupled map lattice formalism in which many hundreds of patches are connected by diffusive dispersal, they demonstrated host-parasitoid persistence is possible and that the resultant dynamics generate complex spatial patterns - spatial chaos, spirals, and crystal lattices - that are essential for persistence. Coexistence was found to be robust to the presence of environmental variability when applied multiplicatively as white noise to the host rate of increase. Interestingly environmental variation did not disrupt the spatial pattern (and coexistence) if it occurred at a smaller scale than dispersal, however, spatial patterns were disrupted when the scale of environmental variation exceeded the dispersal distance of the individual. It was not noted whether other forms of environmental variability (e.g., coloured noise) altered this conclusion, or even reinforced coexistence.

Ultimately, CSS must explicitly study the effects of realistic properties of environmental variation on ecological dynamics [87-92]. For example, it has been recently found that the effects of environmental noise on the temporal stability of food webs composed of complex population oscillations depends upon their amplitude, synchrony and correlation [93]. Gouhier et al. [75] studied the dynamics of the keystone food web motif (a diamond configuration of a predator consuming two prey species competing for a shared resource) in a metacommunity to reveal the interacting effects of environmental variability and dispersal on food web stability at local and regional scales. Irrespective of dispersal rate, strong environmental fluctuations disrupted compensatory prey dynamics and decreased stability by inducing intermittent correlated fluctuations between prey in local food webs. Other work has proposed that the sudden collapse of an ecological system is thought to arise from nonlinear biotic responses to slow environmental change [94]. Little ecological theory has invoked the interaction between self-organization and the environment to explain the dynamics of collapse, but studies suggest that changes in self-organized vegetation patterns may predict sudden shifts in arid and aquatic ecosystems [95]. In physics, predicting the failure of systems under stress such as material 
rupture, and earthquakes is still a major scientific challenge. Simple models of these systems predict a scale-invariant increase in stress until failure that is characterized by a periodic (complex) scaling exponent (e.g., log periodicity). This periodicity predicts the rate at which simple and localized interactions result in catastrophic (i.e., large amplitude) events and eventually to global system collapse. Log periodicity has recently been applied to forest fires [96,97] and collapse dynamics can more generally be applied to wildfires, disease and insect outbreaks, and to desertification $[98,99]$. Empirical studies of forest fires have demonstrated the importance of environmental heterogeneity in time and space for the predicting their onset and propagation [92,100]. Such heterogeneity is not simply challenging the validity of simple CSS models, but requires that we consider cross-scale feedbacks between self-organized properties and the environment [99]. Local connectivity and self-organized clustering of trees can drive fire propagation [92] but at some threshold scale, fire starts to affect weather conditions that can in turn drive fire propagation, resulting in positive feedback (Figure 2). Such thresholds can be found at multiple scales and explain apparent spatial and temporal discontinuities in natural systems. Theoretical and empirical applications of these ideas reveal how environmental variability can provide a synergistic rather than a competing hypothesis to CSS as a cause of ecological complexity.

\section{Conclusions}

The fact that ecological systems are neither completely predictable nor completely random is not surprising as few natural systems are. However, ecological systems have a substantial random or stochastic component and a worthwhile predictable component that is often masked by the stochastic one, which does make them challenging to study. Dealing with this challenge has required a theoretical understanding that makes use of mathematical, statistical and computational phenomena in which the concepts of determinism and randomness, normally thought of as independent processes, are entwined. Deterministic chaos is a classical example of how these concepts can be entwined, and it has led to a paradigm shift in thinking about how the very sensitive dependence on initial conditions often causes long-term unpredictability in (complex) system behavior or attributes, including ecological systems. Self-organization (e.g., self-organized criticality), which pointed to simple computational rules to explain dramatic regime shifts in systems was another example. The application of fractal dimensions added to the repertoire of tools for understanding complex systems, mainly in describing spatial structures that appeared in ecological systems and could not be measured by Euclidean metrics. While a rich set of tools and theories has emerged over the past few decades, ecological complexity cannot be fully studied using the current definitions and methodology offered by CSS. There exists a great potential for the development of CSS through the exploration of the features of ecological systems that make them distinct (diversity, memory, cross-scale interactions, sensitivity to environmental variability). The challenge for ecologists, however, remains to find generalities in system pattern and dynamics to improve understanding and prediction [101]. CSS has a track record of finding such generalities within varied classes of complex systems and in our opinion can be better developed to serve this need of ecology.

\section{Acknowledgements}


This article was born out of an organized oral session entitled "Biocomplexity Scaling: an ecological perspective” at the 2004 Annual Meeting of the Ecological Society of America. The authors thank Andrea Belgrano, Bai-Lian Li, the late Erik Rauch and David Schneider for also presenting papers in the session and earlier discussions. Funding is gratefully acknowledged from NSERC (all co-authors) and the Canada Research Chairs program (MA and AG.).

\section{References}

1. May, R.M. Stability and Complexity in Model Ecosystems; Princeton University Press: Princeton, NJ, USA, 1973; p. 235.

2. Allen, T.F.H.; Starr, T.B. Hierarchy: Perspectives for Ecological Complexity; University of Chicago Press: Chicago, IL, USA, 1982; p. 310.

3. Maurer, B.A. Untangling Ecological Complexity: The Macroscopic Perspective; University of Chicago Press: Chicago, IL, USA, 1999; p. 251.

4. Levin, S.A. Ecosystems and the biosphere as complex adaptive systems. Ecosystems 1998, 1, 431-436.

5. Michener, W.K.; Baerwald, T.J.; Firth, P.; Palmer, M.A.; Rosenberger, J.L.; Sandlin, E.A.; Zimmerman, H. Defining and unraveling biocomplexity. Bioscience 2001, 51, 1018-1023.

6. Rosen, R. Life Itself: A Comprehensive Inquiry into the Nature, Origin, and Fabrication Of Life; Columbia University Press: New York, NY, USA, 1991; p. 285.

7. Collot, F. Correlations entre complexification et instabilite dans une formalisation du concept de complexite. Acta Biotheor. 1995, 43, 195-204.

8. Ahl, V.; Allen, T.F.H. Hierarchy Theory: A Vision, Vocabulary, And Epistemology; Columbia University Press: New York, NY, USA, 1996; p. 206.

9. de Wailly, A. The ambiguity of the word "complexity" a proposal for clarification. Acta Biotheor. 1998, 46, 177-183.

10. Ricard, J. What do we mean by biological complexity? C. R. Biol. 2003, 326, 133-140.

11. Jørgensen, S.E. Integration of Ecosystem Theories: A Pattern, 3rd ed.; Kluwer Academic Publishers: Boston, MA, USA, 2002; p. 420.

12. Horgan, J. From complexity to perplexity. Sci. Amer. 1995, 272, 104-109.

13. Gell-Mann, M.; Lloyd, S. Information measures, effective complexity, and total information. Complexity 1996, 2, 44-52.

14. Langton, C. Life at the edge of chaos. In Artificial Life II; Addison Wesley: New York, NY, USA, 1992; Volume 10, pp. 41-91.

15. Schroeder, M.R. Fractals, Chaos, Power Laws: Minutes From an Infinite Paradise; W.H. Freeman: New York, NY, USA, 1991; p. 429.

16. Brown, J.H.; Gupta, V.K.; Li, B.L.; Milne, B.T.; Restrepo, C.; West, G.B. The fractal nature of nature: power laws, ecological complexity and biodiversity. Phil. Trans. Roy. Soc. B-Biol. Sci. 2002, 357, 619-626.

17 Hubbell, S. The Unified Neutral Theory of Biodiversity and Biogeography; Princeton University Press: Princeton, NJ, USA, 2001; p. 448. 
18. Grimm, V.; Revilla, E.; Berger, U.; Jeltsch, F.; Mooij, W.M.; Railsback, S.F.; Thulke, H.H.; Weiner, J.; Wiegand, T.; DeAngelis, D.L. Pattern-oriented modeling of agent-based complex systems: Lessons from ecology. Science 2005, 310, 987-991.

19. Boccara, N. Modeling Complex Systems; Springer: New York, NY, USA, 2004; p. 397.

20. Bascompte, J.; Sole, R.V. Rethinking complexity-modeling spatiotemporal dynamics in ecology. Trend. Ecol. Evolut. 1995, 10, 361-366.

21. Storch, D.; Gaston, K.J. Untangling ecological complexity on different scales of space and time. Basic Appl. Ecol. 2004, 5, 389-400.

22. O’Dwyer, J.; Green, J. Field theory for biogeography: a spatially explicit model for predicting patterns of biodiversity. Ecol. Lett. 2010, 13, 87-95.

23. Barabasi, A.L.; Albert, R. Emergence of scaling in random networks. Science 1999, 286, 509-512.

24. Albert, R.; Jeong, H.; Barabasi, A.L. Error and attack tolerance of complex networks. Nature 2000, 406, 378-382.

25. Pascual, M.; Dunne, J.A. Ecological Networks: Linking Structure to Dynamics in Food Webs; Oxford University Press: Oxford, NY, USA, 2006; p. 386.

26. Banasek-Richter, C.; Bersier, L.F.; Cattin, M.F.; Baltensperger, R.; Gabriel, J.P.; Merz, Y.; Ulanowicz, R.E.; Tavares, A.F.; Williams, D.D.; De Ruiter, P.C.; Winemiller, K.O.; Naisbit, R.E. Complexity in quantitative food webs. Ecology 2009, 90, 1470-1477.

27. Girvan, M.; Newman, M.E.J. Community structure in social and biological networks. Proc. Nat. Acad. Sci. USA 2002, 99, 7821-7826.

28. Krause, A.E.; Frank, K.A.; Mason, D.M.; Ulanowicz, R.E.; Taylor, W.W. Compartments revealed in food-web structure. Nature 2003, 426, 282-285.

29. Allesina, S.; Pascual, M. Googling Food Webs: Can an eigenvector measure species' importance for coextinctions? Plos Comp. Biol. 2009, 5, 1-6.

30. Dunne, J.A.; Williams, R.J.; Martinez, N.D. Network structure and biodiversity loss in food webs: robustness increases with connectance. Ecol. Lett. 2002, 5, 558-567.

31. Melian, C.J.; Bascompte, J. Complex networks: two ways to be robust? Ecol. Lett. 2002, 5, 705-708.

32. Williams, R.J.; Martinez, N.D. Simple rules yield complex food webs. Nature 2000, 404, 180-183.

33. Rikvold, P.A.; Zia, R.K.P. Punctuated equilibria and 1/f noise in a biological coevolution model with individual-based dynamics. Phys. Rev. E 2003, 68, 31913.

34. McKane, A.; Drossel, B. Modelling evolving food webs. In Dynamical Food Webs; Elsevier: Amsterdam, The Netherlands, 2005; pp. 74-88.

35. Christensen, K.; de Collobiano, S.A.; Hall, M.; Jensen, H.J. Tangled nature: A model of evolutionary ecology. J. Theor. Bio. 2002, 216, 73-84.

36. Filotas, E.; Grant, M.; Parrott, L.; Rikvold, P. The effect of positive interactions on community structure in a multi-species metacommunity model along an environmental gradient. Ecol. Model. 2010, 221, 885-894.

37. Bell, G. Experimental evolution. Heredity 2008, 100, 441-442. 
38. Parrott, L.; Kok, R. A generic primary producer model for use in ecosystem simulation. Ecol. Model. 2001, 139, 75-99.

39. Wilson, W. Simulating ecological and evolutionary systems in C. Cambridge University Press: New York, NY, USA, 2000.

40. Huston, M. A general hypothesis of species diversity. Amer. Naturalist 1979, 113, 81.

41. Levin, S.; Paine, R. Disturbance, patch formation, and community structure. Proc. Nat. Acad. Sci. 1974, 71, 2744.

42. Wiens, J. Population responses to patchy environments. Annu. Rev. Ecol. Syst. 1976, 7, 81-120.

43. Pickett, S.; White, P. The Ecology of Natural Disturbance and Patch Dynamics; Academic Press: Orlando, Florida, USA, 1986.

44. Schlicht, R.; Iwasa, Y. Forest gap dynamics and the Ising model. J. Theor. Biol. 2004, 230, 65-75.

45. Pagnutti, C.; Azzouz, M.; Anand, M. Propagation of local interactions create global gap structure and dynamics in a tropical rainforest. J. Theor. Biol. 2007, 247, 168-181.

46. Wootton, J.T. Local interactions predict large-scale pattern in empirically derived cellular automata. Nature 2001, 413, 841-844.

47. Guichard, F.; Halpin, P.M.; Allison, G.W.; Lubchenco, J.; Menge, B.A. Mussel disturbance dynamics: Signatures of oceanographic forcing from local interactions. Amer. Naturalist 2003, 161, 889-904.

48. Turcotte, D.L.; Malamud, B.D.; Guzzetti, F.; Reichenbach, P. Self-organization, the cascade model, and natural hazards. Proc. Nat. Acad. Sci. U.S.A. 2002, 99, 2530-2537.

49. Pascual, M.; Guichard, F. Criticality and disturbance in spatial ecological systems. Trend. Ecol. Evol. 2005, 20, 88-95.

50. Lawton, J. H. Population Dynamics-More time means more variation. Nature 1988, 334, 563-563.

51. Pimm, S.; Redfearn, A. The variability of natural populations. Nature 1988, 334, 613-614.

52. Inchausti, P.; Halley, J. The long-term temporal variability and spectral colour of animal populations. Evol. Ecol. Res. 2002, 4, 1033-1048.

53. Sole, R.V.; Manrubia, S.C.; Benton, M.J.; Kauffman, S.; Bak, P. Criticality and scaling in evolutionary ecology. Trend. Ecol. Evol. 1999, 14, 156-160.

54. Frigg, R. Self-organised criticality-what it is and what it isn't. Stud. Hist. Philos. Sci. 2003, 34A, 613-632.

55. Kolasa, J. Complexity, system integration, and susceptibility to change: Biodiversity connection. Ecol. Complex. 2005, 2, 431-442.

56. Cohen, J.E. Unexpected dominance of high-frequencies in chaotic nonlinear population-models. Nature 1995, 378, 610-612.

57. Sugihara, G. Ecology-from out of the Blue. Nature 1995, 378, 559-560.

58. Blarer, A.; Doebeli, M. In the red zone. Nature 1996, 380, 589-590.

59. Miramontes, O.; Rohani, P. Intrinsically generated coloured noise in laboratory insect populations. Proc. Roy. Soc. London Ser. B. 1998, 265, 785-792.

60. Kaitala, V.; Ranta, E. Red/blue chaotic power spectra. Nature 1996, 381, 198-199. 
61. White, A.; Begon, M.; Bowers, R.G. Explaining the colour of power spectra in chaotic ecological models. Proc. Roy. Soc. London Ser. B. 1996, 263, 1731-1737.

62. Taylor, P.J. Unruly Complexity: Ecology, Interpretation, Engagement; University of Chicago Press: Chicago, IL, USA, 2005; p. 289.

63. Anand, M.; Orloci, L. Complexity in plant communities: The notion and quantification. J. Theor. Biol. 1996, 179, 179-186.

64. Dale, M.B.; Anand, M.; Desrochers, R.E. Measuring information-based complexity across scales using cluster analysis. Ecol. Inform. 2007, 2, 121-127.

65. Li, S.C.; Chang, Q.; Peng, F.A.; Wang, Y.L. Indicating landscape fragmentation using L-Z complexity. Ecol. Indic. 2009, 9, 780-790.

66. Proulx, R.; Parrott, L. Measures of structural complexity in digital images for monitoring the ecological signature of an old-growth forest ecosystem. Ecol. Indic. 2008, 8, 270-284.

67. McCann, K.S. The diversity-stability debate. Nature 2000, 405, 228-233.

68. Borda-de-Agua, L.; Hubbell, S.P.; McAllister, M. Species-area curves, diversity indices, and species abundance distributions: A multifractal analysis. Amer. Naturalist 2002, 159, 138-155.

69. Ricotta, C.; Anand, M. Spatial complexity of ecological communities: Bridging the gap between probabilistic and non-probabilistic uncertainty measures. Ecol. Model. 2006, 197, 59-66.

70. Luque, B.; Ballesteros, F.J.; Fernandez, M. Variances as order parameter and complexity measure for random Boolean networks. J. Phys.a-Math. Gen. 2005, 38, 1031-1038.

71. Murphy, H.T.; Lovett-Doust, J. Context and connectivity in plant metapopulations and landscape mosaics: does the matrix matter? Oikos 2004, 105, 3-14.

72. Demetrius, L.; Gundlach, V.M.; Ochs, G. Complexity and demographic stability in population models. Theor. Pop. Biol. 2004, 65, 211-225.

73. Scheffer, M.; Bascompte, J.; Brock, W.A.; Brovkin, V.; Carpenter, S.R.; Dakos, V.; Held, H.; van Nes, E.H.; Rietkerk, M.; Sugihara, G. Early-warning signals for critical transitions. Nature 2009, 461, 53-59.

74. Rai, V.; Anand, M.; Upadhyay, R.K. Trophic structure and dynamical complexity in simple ecological models. Ecol. Complex. 2007, 4, 212-222.

75. Gouhier, T.C.; Guichard, F.; Gonzalez, A. Synchrony and stability of food webs in metacommunities. Amer. Naturalist 2010, 175, E16-E34.

76. Hendry, R.J.; Mcglade, J.M. The Role of Memory in Ecological-Systems. Proc. Roy. Soc. Lon. Ser. B 1995, 259, 153-159.

77. Padisak, J. Seasonal succession of phytoplankton in a large shallow lake (Balaton, Hungary)—a dynamic approach to ecological memory, its possible role and mechanisms. J. Ecol.1992, 80, 217-230.

78. Hairston, N.G.; Lampert, W.; Caceres, C.E.; Holtmeier, C.L.; Weider, L.J.; Gaedke, U.; Fischer, J.M.; Fox, J.A.; Post, D.M. Lake ecosystems-Rapid evolution revealed by dormant eggs. Nature 1999, 401, 446-446.

79. Peterson, G.D. Contagious disturbance, ecological memory, and the emergence of landscape pattern. Ecosystems 2002, 5, 329-338.

80. Schwinning, S.; Sala, O.E.; Loik, M.E.; Ehleringer, J.R. Thresholds, memory, and seasonality: understanding pulse dynamics in arid/semi-arid ecosystems. Oecologia 2004, 141, 191-193. 
81. Milinski, M. Long-term memory for food patches and implications for Ideal Free Distributions in sticklebacks. Ecology 1994, 75, 1150-1156.

82. Hirvonen, H.; Ranta, E.; Rita, H.; Peuhkuri, N. Significance of memory properties in prey choice decisions. Ecol. Model. 1999, 115, 177-189.

83. Ashby, W.R. An Introduction to Cybernetics; J. Wiley: New York, NY, USA, 1956; p. 295.

84. Golinski, M.; Bauch, C.; Arland, M. The effects of endogenous ecological memory on population stability and resilience in a variable environment. Ecol. Model. 2008, 212, 334-341.

85. Grimm, V.; Railsback, S.F. Individual-Based Modeling and Ecology; Princeton University Press: Princeton, NJ, USA, 2005; p. 428.

86. Hassell, M.; Godfray, H.; Comins, H. Effects of global change on the dynamics of insect host-parasitoid interactions. Biotic Interaction. Glob. Change 1993, 402-423.

87. Halley, J. M. Ecology, evolution and 1/f-noise. Trend. Ecol. Evolut. 1996, 11, 33-37.

88. Ripa, J.; Ives, A.R. Food web dynamics in correlated and autocorrelated environments. Theor. Pop. Biol. 2003, 64, 369-384.

89. Ripa, J.; Lundberg, P.; Kaitala, V. A general theory of environmental noise in ecological food webs. Amer. Naturalist 1998, 151, 256-263.

90. Gonzalez, A.; De Feo, O. Environmental variability modulates the insurance effects of diversity in nonequilibrium communities. In The Impact of Environmental Variability on Ecological Systems; Vasseur, D.A., McCann, K.S., Eds.; Springer: Dordrecht, The Netherlands, 2007; pp. 133-158.

91. Gonzalez, A.; Holt, R.D. The inflationary effects of environmental fluctuations in source-sink systems. Proc. Nat. Acad. Sci. USA 2002, 99, 14872-14877.

92. Turner, M.; Romme, W.; Tinker, D. Surprises and lessons from the 1988 Yellowstone fires. Fron. Ecol. Environ. 2003, 1, 351-358.

93. Vasseur, D.A.; Fox, J.W. Environmental fluctuations can stabilize food web dynamics by increasing synchrony. Ecol. Lett. 2007, 10, 1066-1074.

94. Scheffer, M.; Carpenter, S.; Foley, J.A.; Folke, C.; Walker, B. Catastrophic shifts in ecosystems. Nature 2001, 413, 591-596.

95. Rietkerk, M.; Dekker, S.C.; de Ruiter, P.C.; van de Koppel, J. Self-organized patchiness and catastrophic shifts in ecosystems. Science 2004, 305, 1926-1929.

96. Sornette, D. Critical Phenomena in Natural Sciences: Chaos, Fractals, Self Organization, and Disorder: Concepts and Tools, 2nd ed.; Springer: Berlin, NY, USA, 2003; p. 528.

97. Malamud, B.D.; Morein, G.; Turcotte, D.L. Log-periodic behavior in a forest-fire model. Nonlinear Process. Geophys. 2005, 12, 575-585.

98. Ferriere, R.; Cazelles, B. Universal power laws govern intermittent rarity in communities of interacting species. Ecology 1999, 80, 1505-1521.

99. Peters, D.P.C.; Pielke, R.A.; Bestelmeyer, B.T.; Allen, C.D.; Munson-McGee, S.; Havstad, K.M. Cross-scale interactions, nonlinearities, and forecasting catastrophic events. Proc. Nat. Acad. Sci. U.S.A. 2004, 101, 15130-15135.

100. Whelan, R.J. The Ecology of Fire; Cambridge University Press: Cambridge, NY, USA, 1995; p. 346. 
101. Coreau, A.; Pinay, G.; Thompson, J.D.; Cheptou, P.O.; Mermet, L. The rise of research on futures in ecology: rebalancing scenarios and predictions. Ecol. Lett. 2009, 12, 1277-1286.

(C) 2010 by the authors; licensee Molecular Diversity Preservation International, Basel, Switzerland. This article is an open-access article distributed under the terms and conditions of the Creative Commons Attribution license (http://creativecommons.org/licenses/by/3.0/). 